\title{
Atividade inseticida das plantas e aplicações: revisão
}

CORRÊA, J.C.R.*; SALGADO, H.R.N.

Departamento de Fármacos e Medicamentos, Faculdade de Ciências Farmacêuticas, Universidade Estadual Paulista, UNESP, Rodovia Araraquara-Jaú, km 1, CEP: 14801-902, Araraquara-Brasil *josilenechavescorrea @gmail.com

\begin{abstract}
RESUMO: A utilização de agrotóxicos tem contribuído para o aumento da produção agrícola, entretanto, o uso incorreto e indiscriminado durante várias décadas levou à acumulação de resíduos tóxicos em alimentos, contaminação da água e do solo, intoxicação de produtores rurais, seleção de pragas resistentes, entre muitos outros problemas. Ultimamente tem crescido o interesse por substâncias que apresentem menor risco à saúde humana e ao ambiente, além da demanda crescente por produtos alimentícios saudáveis e isentos de resíduos de agrotóxicos. Felizmente são inúmeras as plantas que apresentam atividade inseticida, devendo ser estudadas e introduzidas, quando possível, nas propriedades agrícolas como forma alternativa de controle de pragas. Neste trabalho, é apresentada revisão sobre o uso de plantas com propriedades inseticidas e repelentes, evidenciando o potencial dessa ferramenta no manejo de pragas. Para a inserção definitiva e segura de produtos botânicos no mercado, mais estudos ainda são necessários.
\end{abstract}

Palavras-chave: plantas, atividade inseticida, ação repelente

ABSTRACT: Insecticidal activities of plants and applications: a review. The use of pesticides has contributed to the increased agricultural production; however, the incorrect and indiscriminate use over several decades has led to the accumulation of toxic residues in food, contamination of water and soil, poisoning of farmers, selection of resistant pests, besides several other problems. Lately, the interest for substances posing lower risk to the human health and the environment has increased, in addition to the increasing demand for healthy food products free from pesticide residues. Fortunately a large number of plants have insecticidal activity and should be studied and introduced, whenever possible, into farms as an alternative means to control pests. In this study, a review of the use of plants with insecticidal or repellent potential is presented, evidencing the potential of this tool in pest management. For a definitive and safe insertion of botanical products into the market further studies are still needed.

Key words: plants, insecticidal activity, repellent action

O crescimento da população mundial levou ao aumento de demandas de produção de alimentos (Menezes, 2005) e a diminuição da população em áreas rurais e das fazendas (Carlini \& Grossi-de-Sá, 2002) tornou este problema mais difícil. Para satisfazer à demanda cada vez maior de alimentos, uma revolução verde, datada da década de 1960, levou a agricultura a se caracterizar como monoculturas extensivas e de grande utilização de fertilizantes químicos sintéticos e agrotóxicos (Menezes, 2005).

Apesar da utilização dessas substâncias terem contribuído para o aumento da produção agrícola, trouxe também muitos males. O uso incorreto e indiscriminado durante várias décadas levou ao acúmulo de resíduos tóxicos em alimentos, contaminação da água e do solo, intoxicação de produtores rurais, aparecimento de pragas resistentes, o interrompimento do sistema de controle biológico por inimigos naturais, ocasionando surtos de insetospraga, entre muitos outros problemas (Kim et al., 2003; Costa et al., 2004; Menezes, 2005). O brometo de metila, por exemplo, agente muito eficaz para a proteção de alimentos armazenados, é um potencial destruidor da camada de ozônio (Kim et al., 2003).

Os inseticidas botânicos foram muito populares e importantes entre as décadas de 30 e 40 e o Brasil foi grande produtor e exportador destes produtos, substâncias como piretro, rotenona e nicotina, que apresentam maior segurança no uso agrícola e menor impacto ambiental (Menezes, 2005).

Recebido para publicação em 06/04/2010

Aceito para publicação em 11/07/2009

Rev. Bras. PI. Med., Botucatu, v.13, n.4, p.500-506, 2011. 
Os inseticidas naturais foram gradativamente substituídos pelos sintéticos, pois apresentavam problemas como variações na eficiência, devido a diferenças na concentração do ingrediente ativo entre plantas e baixo efeito residual, que obrigava a se fazer várias aplicações em períodos curtos (Costa et al., 2004).

Ultimamente o interesse pelos produtos botânicos para o controle de pragas tem aumentado. Substâncias com menores riscos à saúde humana e ao ambiente vem sendo avaliadas, fato este somado à demanda crescente por produtos alimentícios saudáveis e isentos de resíduos de agrotóxicos. Os problemas decorrentes da utilização de pesticidas químicos apontam para a necessidade de se desenvolver novos tipos de agentes de controle mais seletivos e menos agressivos ao homem e ambiente (Kim et al., 2003; Menezes, 2005).

Felizmente, são inúmeras as plantas possuidoras de atividade inseticida, e muitas precisam ser estudadas e introduzidas, quando possível, nas propriedades agrícolas como forma alternativa de controle de pragas (Menezes, 2005). As plantas são ricas em substâncias bioativas, que são, frequentemente, ativas contra número limitado de espécies. Algumas não específicas, muitas vezes são biodegradáveis e apresentam baixa ou nenhuma toxicidade a mamíferos. Assim, o estudo pode acarretar o desenvolvimento de novas classes de agentes de controle mais seguras (Kim et al., 2003).

Neste trabalho a utilização de plantas com atividade inseticida ou repelente é abordada, incluindo as mais recentes pesquisas nesta área. Extensa pesquisa foi realizada nas bases de dados Scopuse SciFinder com os unitermos plants AND insecticidal action AND bioinsecticides AND repellents botanic inseticides.

Inseticidas botânicos são compostos resultantes do metabolismo secundário das plantas (Kim et al., 2003), que compõem a própria defesa química contra os insetos herbívoros. Os princípios ativos inseticidas podem derivar de toda a planta ou partes dela, podem ser o próprio material vegetal, normalmente, moído até ser reduzido a pó, ou produtos derivados por extração aquosa ou com solventes orgânicos (Menezes, 2005).

Algumas substâncias botânicas têm atividade inseticida conhecida, tais como, piretrinas, rotenona, nicotina, cevadina, veratridina, rianodina, quassinoides, azadiractina e biopesticidas voláteis. Estes últimos são, normalmente, óleos essenciais presentes nas plantas aromáticas (Isman, 2000).

As mais recentes investigações em vários países confirmam que alguns óleos essenciais de plantas têm não apenas a capacidade de repelir insetos, mas apresentam também ação inseticida através do contato direto ou pelas vias respiratórias dos insetos. Alguns apresentam ainda ação fungicida contra alguns patógenos importantes de plantas (Isman, 2000). Um exemplo de óleo essencial com ação inseticida é o óleo da citronela, presente em algumas plantas aromáticas, como o capim limão (Cymbopogon citratus, Poaceae) e o eucalipto citriodora (Eucaliptus citriodora), sendo utilizado para a fabricação de repelentes contra mosquitos e borrachudos (Menezes, 2005).

Deve-se ter cuidado ao utilizar óleos essenciais botânicos ou seus constituintes, pois aqueles que apresentam alta eficácia podem ser também os mais fitotóxicos (Isman, 2000). A atividade tóxica sobre plantas pode retardar o desenvolvimento e diminuir a produtividade, ou até levar o vegetal à morte. $O$ aparecimento dos efeitos fitotóxicos depende da forma como as substâncias são aplicadas e da dose empregada.

São necessários mais estudos sobre os efeitos fitotóxicos dos inseticidas botânicos, como o apresentado por Rani \& Murthy (2008) que mostraram que a capacidade de germinação das sementes de milho tratadas com inseticidas provenientes das plantas Piper cubeba L., Coriandrum sativum L., Eichhornia crassipes Mart., Limonia acidissima L, Tamarindus indica L., Cocos nucifera L., Terminalia catappa L., Syzygium cumini L. e Coccinia indica permaneceu inalterada.

Certamente, se as propriedades fitotóxicas de algum óleo essencial forem acentuadas, esta característica pode ser utilizada para o desenvolvimento de um herbicida botânico. Alguns dos componentes de óleos essenciais, quando puros, podem apresentar toxicidade a mamíferos. $O$ carvacrol apresenta $\mathrm{DL}_{50}$ de 2-3 $\mathrm{g} \mathrm{kg}^{-1}$ para ratos. No entanto, os óleos essenciais são uma mistura de várias substâncias, e quando testado o óleo essencial que contém o carvacrol na dosagem de $2 \mathrm{~g} \mathrm{~kg}^{-1}$ não houve mortalidade em ratos (Isman, 2000).

Tem sido demonstrada a atividade repelente e a importância dos efeitos sinérgicos entre os componentes dos óleos essenciais, que são misturas voláteis de hidrocarbonetos com diversidade de grupos funcionais. Entre as famílias de plantas promissoras, com óleos essenciais utilizados para repelir insetos, Cymbopogon spp., Ocimum spp. e Eucalyptus spp. são as mais citadas. Alguns dos compostos presentes nestas misturas incluem cânfora-pineno, limoneno, citronelol, citronelal e timol (Nerio et al., 2009a).

Apesar de haver informações sobre grande número de plantas com atividade inseticida, fungicida e efeito nematicida, ainda falta o adequado desenvolvimento de produtos que possam ser disponibilizados comercialmente (Vasantharaj, 2008).

O repelente sintético DEET ( $\mathrm{N}, \mathrm{N}$-dietil-mmetilbenzamida) é muito utilizado topicamente e altamente eficaz contra mosquitos e artrópodes causadores de doenças. No entanto, intoxicações 
foram relatadas em algumas circunstâncias, especialmente em crianças e idosos. Outra limitação deste repelente é a baixa eficácia contra os principais vetores de parasitas da malária humana, Anopheles spp. Além disso, já foi documentada a existência de mutante de Drosophila melanogaste insensível ao DEET (Paluch et al., 2009).

O uso de plantas como repelentes contra insetos é comum, como mostrado por Kweka et al. (2008) inclusive contra vetores da malária. Desta forma, é necessário investigar o potencial destas plantas para o controle de insetos (Kweka et al., 2008). Apesar de, financeiramente, os produtos sintéticos ainda serem vantajosos e extensamente utilizados, os produtos naturais têm o potencial de proporcionar repelência eficiente e mais segura ao homem e ambiente.

Apesar das vantagens declaradas, como a ação e degradação rápidas, toxicidade baixa a moderada para mamíferos, maior seletividade e baixa fitotoxidade, os inseticidas botânicos apresentam algumas desvantagens como necessidade de utilização de composto sinergista, baixa persistência, carência de pesquisas, escassez do recurso natural, necessidade de padronização química e controle de qualidade, dificuldade de registro e custo. Além disso, a falta de dados relacionados à fitotoxicidade, à persistência e aos efeitos sobre organismos benéficos $\mathrm{e}$ as dificuldades relacionadas ao isolamento de princípios ativos e a concentração em diferentes partes vegetais, também são algumas barreiras a serem rompidas e mais estudos nesta área são necessários (Isman, 2000; Costa et al., 2004; Menezes, 2005).

Os princípios ativos dos inseticidas botânicos são normalmente compostos por um complexo conjunto de substâncias. Para registro de produto inseticida no órgão competente é necessária a identificação de todas as substâncias ativas e a realização de testes toxicológicos. Portanto, tornase difícil e custoso o registro de produtos preparados a partir de plantas (Menezes, 2005).

Podem atuar de várias formas, principalmente, quando o responsável pela ação é uma complexa mistura de substâncias. Os metabólicos secundários de plantas com efeitos inseticidas podem agir como inibidores da alimentação de insetos ou dificultadores de crescimento, desenvolvimento, reprodução e comportamento (Menezes, 2005).

Os inseticidas botânicos podem apresentar ação tóxica e causar a morte de insetos, atuando sobre o sistema nervoso central. É importante notar que muitas substâncias que apresentam ação tóxica aos insetos, podem apresentar também efeitos similares no homem (Menezes, 2005).

Algumas substâncias agem como repelentes, impedindo que os insetos se aproximem das plantas.
Há, também, os inseticidas que atuam como agente antialimentar, impedindo que os insetos iniciem a alimentação, causando morte por inanição. As substâncias que atuam por ingestão, penetram no organismo por via oral, que é uma forma específica de atuação, restrita a insetos herbívoros, apresentando, portanto, pouca toxicidade a humanos (Menezes, 2005).

Muitos insetos têm a capacidade de se alimentarem de plantas que contêm substâncias tóxicas, sem serem prejudicados utilizando, principalmente, mecanismos enzimáticos para inativação dos princípios ativos ou evitando os estágios e as partes da planta onde as substâncias estejam presentes em maior concentração. Nestes casos, devem-se utilizar extratos com tais substâncias em maior concentração, inibindo a alimentação desses insetos ou prejudicando-os após a ingestão (Costa et al., 2004).

Outro mecanismo de ação envolve atividade sobre órgão ou molécula alvo. Neste caso, atuam dificultando o crescimento e o desenvolvimento, interferindo no metabolismo celular (Menezes, 2005). Dependendo da concentração utilizada, alguns extratos podem reduzir a viabilidade de ovos, ninfas, larvas e pupas. A redução do número de ovos e a inibição da oviposição são importantes efeitos de extratos vegetais sobre a reprodução dos insetos (Costa et al., 2004).

Outras substâncias atuam por contato, ou seja, agem e são absorvidas pela quitina e exoesqueleto ou pelas vias respiratórias (ação fumigante), podem ser úteis para o controle de pragas que atacam alimentos em armazéns e silos.

A literatura apresenta muitos trabalhos publicados que avaliam a atividade inseticida de compostos botânicos apresentando resultados expressivos (Tabela 1).

Kim et al. (2003) relatam atividade inseticida de cinco óleos essenciais e extratos de 30 plantas aromáticas sobre os carunchos de feijão Callosobruchus chinensis (L.) e de arroz Sitophilus oryzae (L.). Óleos essenciais de alho (Allium scorodoprasm), pimenta (Capsicum annum), rabanete (Cocholeria aroracia), mostarda (Brassica juncea) e de canela (Cinnamomum cassia) e extratos metanólicos das 30 diferentes plantas foram testados pelo contato direto, aplicando-os sobre papéis de filtro que, após secos, foram introduzidos em um frasco contendo 20 indivíduos adultos provenientes de culturas laboratoriais destes insetos.

Para o caruncho de arroz, os óleos essenciais de rabanete, mostarda e canela provocaram $100 \%$ de mortalidade após o primeiro dia de tratamento. O mesmo foi observado para o extrato de canela. Algumas plantas apresentaram resultados consideráveis entre o segundo e o quarto dia de

Rev. Bras. Pl. Med., Botucatu, v.13, n.4, p.500-506, 2011. 
tratamento como Cinnamomum sieboldii (100\% no segundo dia), Acorus calamus var. angustatus, Acorus gramineus, Agastache rugosa e Foeniculum vulgare (100\% no terceiro dia); além de lllicium verum que apresentou $100 \%$ de mortalidade no quarto dia. Em uma dose cinco vezes inferior, os óleos essenciais de mostarda, canela e rabanete resultaram em 100\% de mortalidade dos insetos, no primeiro dia de tratamento; extratos de Acorus calamus var. angustatus, Cinnamomum cassia, Cinnamomum sieboldi acarretaram $100 \%$ de mortalidade, no terceiro dia, extratos de Acorus gramineus e Foeniculum vulgare, $100 \%$ no quarto dia.

Para o caruncho de feijão, os extratos de Acorus calamus var. angustatus, Eugenia caryophyllata, Foeniculum vulgare, Illicium verum, Aquillaria agallocha, Cinnamomum cassia, Thymus mandschuricus, Cinnamomum sieboldii, e os óleos essenciais de alho, mostarda e canela acarretaram $100 \%$ de mortalidade dos insetos no primeiro dia de tratamento. Em uma dose cinco vezes menor, a utilização dos extratos de Cinnamomum cassia, Cinnamomum sieboldi, Illicium verum e os óleos essenciais de canela e rabanete provocaram $100 \%$ de mortalidade dos insetos no primeiro dia de tratamento, enquanto o óleo essencial de alho e o extrato de Foeniculum vulgare acarretaram $100 \%$ de mortalidade no segundo dia.

Os óleos essenciais de rabanete, mostarda e canela foram testados quanto à ação fumigante. Todos resultaram em $100 \%$ de mortalidade quando testados pelos métodos de contato direto ou vapor em recipientes fechados, mas em recipientes abertos, nos dois métodos, os resultados foram muito pequenos ou nulos. Esses resultados confirmam ação fumigante e a possível indicação para controle de pragas em armazéns.

Rathi et al. (2008) realizaram triagem fitoquímica de dez plantas com atividade inseticida, entre elas, Adathoda vasica, Cynodon dactylon, Eclipta alba, Morinda pubescens, Ocimum tenuiflorum, Phyllanthus amarus, Sesbania grandifolora, Solanum surattense, Solanum trilobatume Vinca rosea. Várias misturas de solventes foram utilizadas como extrator. Foram encontrados taninos, importante classe de compostos que agem como uma barreira a insetos herbívoros, e flavonoides na maior parte das plantas estudadas.

Murugesan \& Murugesh (2008) testaram, também, dez extratos diferentes de Azadirachta indica (extrato das folhas de Nim), extrato das folhas de Calotropis gigantea, extrato das folhas de Lantana camara, torta de Nim, óleo de Nim, Nimbecidine $\AA$, extrato de folhas de Pongamia glabra, extrato de folhas de Prosopis juliflora L., extrato de folhas de Vitex negundo, e extrato de alho, Allium sativum. $\mathrm{O}$ teste revelou redução da população de $H$. vigintioctopunctata (besouro Hadda) entre 87,86 e $71,97 \%$ após o terceiro dia de pulverização, sendo que o melhor resultado foi conseguido com o óleo de Nim. Entretanto a eficácia dos extratos foi reduzida ao passar dos dias após a pulverização.

Irulandi et al. (2008) relatam diferenças entre a atividade inseticida apresentada por extratos botânicos contra a broca do café, Hypothenemus hampei, em estudos laboratoriais e estudos no campo. O extrato de Nim, por exemplo, apresentou maior atividade inseticida quando testado em laboratório do que quando testado nas plantações. Segundo os autores os resultados discrepantes podem ser explicados pela ação solar sobre os extratos levando a fotodegradação.

Os óleos essenciais de Ocimum suave e Ocimum kilimandscharicum foram avaliados contra vetores da malária no nordeste da Tanzânia Oriental (Kweka et al., 2008). O estudo etnobotânico foi conduzido em Moshi, na região nordeste do Kilimanjaro, na Tanzânia. Através de entrevistas, investigaram espécies de plantas utilizadas popularmente como repelentes de insetos. Os bioensaios foram utilizados para avaliar o potencial de proteção de extratos das plantas selecionadas contra mosquitos. As espécies citadas por serem usadas como repelente à noite foram $O$. suave e $O$. kilimandscharicum (Lamiaceae), Azadirachta indica (Meliaceae), Eucalyptus globulus (Myrtaceae) e Lantana camara (Verbenaceae). Os repelentes mais populares foram $O$. kilimandscharicum e $O$. suave, utilizados por $67 \%$ do total de 120 famílias entrevistadas.

Em bioensaio, com óleos essenciais das duas plantas de Ocimum, foi comparado com citronela e DEET para estudar a repelência e inibição da alimentação dos insetos em braços de voluntários. Usando papéis de filtro impregnados com extratos de Ocimum, efeitos 'knockdown' e a mortalidade foram investigados sobre os mosquitos da malária Anopheles arabiensis e Anopheles gambiae, e ainda o mosquito, Culex quinquefasciatus. Alta proteção contra picadas (inibição de 83 a 91\%) e alimentação $(71,2$ a $92,5 \%)$ foi observada para as três espécies de mosquitos. Os autores afirmam que o uso de plantas inteiras e seus produtos como repelentes de insetos é comum entre as comunidades rurais do nordeste da Tanzânia Oriental e os resultados indicam que o uso de $O$. suave e $O$. kilimandscharicum como repelentes seria benéfico na redução do vetor da malária (Kweka et al., 2008).

Atualmente, o uso de produtos químicos sintéticos para o controle de artrópodes suscita várias preocupações relacionadas ao ambiente e à saúde humana e animal. Uma alternativa é a utilização de produtos naturais eficientes e ecologicamente corretos. Entre os produtos naturais, óleos essenciais de plantas pertencentes a várias espécies têm sido

Rev. Bras. PI. Med., Botucatu, v.13, n.4, p.500-506, 2011. 
TABELA 1. Atividade inseticida de compostos botânicos descrita na literatura.

\begin{tabular}{|c|c|c|}
\hline Composto ou espécie botânica & Atividade / praga / hospedeiro & Referência \\
\hline Pisum sativum & $\begin{array}{l}\text { Inseticida/ Sitophilus sp. / grãos estocados } \\
\text { em geral }\end{array}$ & Da Silva et al., 2010 \\
\hline $\begin{array}{l}\text { Cinnamonum cassia, Eugenia caryophyllatae } \\
\text { Pogostemon cablin }\end{array}$ & Inseticida / Dermatophagoides farina / homem & Wu et al., 2010 \\
\hline $\begin{array}{l}\text { Terpenos ( } \alpha \text {-terpineol, carvacrol, linalol, mentol e } \\
\text { timol), }\end{array}$ & Inseticida / Periplaneta americana/ homem & Tong \& Coats, 2010 \\
\hline Iponea carnea, Pedalium nurexe Adhatoda vasica & Inseticida/ Spodoptera litura/ plantas em geral & Sujatha et al., 2010 \\
\hline Monoterpenóides (eugenole cinamaldeído) & $\begin{array}{l}\text { Inseticida / Callosobruchus maculatus (F.) e } \\
\text { Sitophilus oryzae (L.) / grãos estocados em } \\
\text { geral }\end{array}$ & Islam et al., 2010 \\
\hline Ageratum conyzoides & $\begin{array}{l}\text { Inseticida / Sitophilus oryzae / grãos } \\
\text { estocados em geral }\end{array}$ & Novo et al., 2010 \\
\hline Sida acuta & $\begin{array}{l}\text { Inseticida e repelênda / Culex } \\
\text { quinquefasciatus, Aedes aegyptie Anopheles } \\
\text { stephensi / homem }\end{array}$ & Govindarajan, 2010 \\
\hline $\begin{array}{l}\text { Annona squamosa, Capsicum frutescence, Allium } \\
\text { sativum, Zingiber. officinalc, Azadircata indica e } \\
\text { Nicotiana tabacum }\end{array}$ & $\begin{array}{l}\text { Pesticida / C. tomentosicollise M. vitrata / } \\
\text { Vigna unguiculata (L.). Walp. }\end{array}$ & Ahmed et al., 2009 \\
\hline $\begin{array}{l}\text { Amyris (Amyris balsamifera) e Siam-madeira } \\
\text { (Fokienia hodginsii) }\end{array}$ & Repelência / Aedes aegypti / Homem & Paluch et al., 2009 \\
\hline $\begin{array}{l}\text { Lippia origanoides, Lippia alba, Tagetes lúcida, } \\
\text { Rosmarinus officinalis L., Cananga odorata, } \\
\text { Eucalyptus citriodora, e Cymbopogon citratus }\end{array}$ & $\begin{array}{l}\text { Repelência / Sitophilus zeamais (Coleoptera: } \\
\text { Curculionidae)/ Zea mays }\end{array}$ & Nerio et al., $2009 \mathrm{~b}$ \\
\hline $\begin{array}{l}\text { Corymbia citriodora, Eucalyptus camaldulensis, } \\
\text { Ocimum suave e Ocimum basilicum }\end{array}$ & $\begin{array}{l}\text { Repelência / Anopheles arabiensise A. } \\
\text { pharoensis / homem }\end{array}$ & Dugassa et al., 2009 \\
\hline $\begin{array}{l}\text { Syzygium aromaticum, Melaleuca alternifolia, } \\
\text { Zingiber cassamunar, Eucalyptus globules, } \\
\text { Pelargonium graveolens, Boesenbergia pandurata, } \\
\text { Citrus aurantium, Citrus hystrix, Curcuma longa, } \\
\text { Cymbopogon nardus, Rosemarinus oftinalis, } \\
\text { Styrax torkinensis, Vetiveria zizaniodes }\end{array}$ & $\begin{array}{l}\text { Repelência / Leptotrombidium imphalum / } \\
\text { homem }\end{array}$ & Eamsobhana et al., 2009 \\
\hline Ocimum kilimandscharicum e Ocimum suave & $\begin{array}{l}\text { Repelência / Anopheles arabiensis, Anopheles } \\
\text { gambiae e Culex spp. / homem }\end{array}$ & Kweka et al., 2008 \\
\hline $\begin{array}{l}\text { Azadirachta indica, Calotropis gigantean, Lantana } \\
\text { camera, Pongamia glabra, Prosopis juliflora, Vitex } \\
\text { negundo e Allium sativum }\end{array}$ & $\begin{array}{l}\text { Inseticida/ H. vigintioctopunctata/ Solanum } \\
\text { melongena }\end{array}$ & $\begin{array}{l}\text { Murugesan \& Murugesh, } \\
2008\end{array}$ \\
\hline $\begin{array}{l}\text { Azadirachta indica, Acorus calamus, Vitex } \\
\text { negundo, Pongamia glabra, Madhuca latifolia }\end{array}$ & $\begin{array}{l}\text { Inseticida / Hypothenemus hampei(F.) / } \\
\text { Coffea spp. }\end{array}$ & Irulandi et al., 2008 \\
\hline Azadirachta indica & $\begin{array}{l}\text { Inseticida/ Coccidohystrix insolituse } \\
\text { Epilachna vigintioctopunctata Fab. / } \\
\text { Ashwagandha ou Asgandh (Withania } \\
\text { somnifera Dunal) }\end{array}$ & Ravikumar et al., 2008 \\
\hline Argemone mexicana & $\begin{array}{l}\text { Inseticida/ Spodoptera litura Fab. (Noctuidae: } \\
\text { Lepidoptera)/ mais de } 120 \text { hospedeiros }\end{array}$ & Malarvannan et al., 2008 \\
\hline Azadirachta indica & $\begin{array}{l}\text { Inseticida / Pericallia ricini / Riccinus } \\
\text { communis }\end{array}$ & Mala \& Muthalagi, 2008 \\
\hline $\begin{array}{l}\text { Centaurium erythraea, Peganum harmala, } \\
\text { Ajugaiva, Aristolochia bactica, Pteridium aquilinum, } \\
\text { Raphanus raphanistrum }\end{array}$ & $\begin{array}{l}\text { Inseticida / Tribolium castaneum / grãos em } \\
\text { geral }\end{array}$ & Jbilou et al., 2008 \\
\hline $\begin{array}{l}\text { Silene macroserene, Echinopssp., Ostostegia } \\
\text { integrifolia e Olea europaea }\end{array}$ & Repelência / Anopheles arabiensis / homem & Karunamoorthi et al., 2008 \\
\hline
\end{tabular}


TABELA 1. Atividade inseticida de compostos botânicos descrita na literatura.

\begin{tabular}{llc}
\hline \multicolumn{1}{c}{ Composto ou espécie botânica } & \multicolumn{1}{c}{ Atividade / praga / hospedeiro } & Referência \\
\hline Annona coriacea & $\begin{array}{l}\text { Inseticida / Anagasta kuehniella e Corcyra } \\
\text { cephalonica / farinha e Oryza sativa (arroz) }\end{array}$ & Coelho et al., 2007 \\
\hline $\begin{array}{l}\text { Cymbopogon citratus, Attalea princeps e Mentha } \\
\text { arvensis }\end{array}$ & $\begin{array}{l}\text { Repelência / Anopheles darlingi, Mansonia } \\
\text { indubitans e Mansonia titillans / homem }\end{array}$ & Moore et al., 2007 \\
\hline $\begin{array}{l}\text { Sophora flavescens } \\
\text { Origanum majorama, Ocimum basilicum, Salvia } \\
\text { officinalis }\end{array}$ & $\begin{array}{l}\text { Inseticida (antialimentar) / Coptotermes } \\
\text { formosanus / árvores e estruturas de madeira }\end{array}$ & Mao \& Henderson, 2007 \\
\hline $\begin{array}{l}\text { Streblus asper } \\
\text { geral }\end{array}$ & $\begin{array}{l}\text { Inseticida / Dysdercus cingulatus / plantas em } \\
\text { geral }\end{array}$ & Pavela, 2004 Hashim \& Devi, 2003 \\
\hline $\begin{array}{l}\text { Chenopodium multifidum ; Flaeria bidentis; } \\
\text { Aristolochia argentina e Tagetes erecta }\end{array}$ & \begin{tabular}{l} 
Inseticida / Sitophilus oryzae / Zea Mays \\
\hline $\begin{array}{l}\text { Hyptis suaveolens, Daniellia oliveri, Elaeis } \\
\text { guineensis, Azadirachta indica, Eucalyptus sp., } \\
\text { Ocimum canum e Senna occidentalis }\end{array}$
\end{tabular} $\begin{array}{l}\text { Repelência / Anopheles gambiae, An. } \\
\text { pharoensis, Aedes spp., Culex spp., Mansonia } \\
\text { spp. / homem }\end{array}$ & Balsson \& Jaenson, 1999 \\
\hline
\end{tabular}

extensivamente testados para avaliar as propriedades repelentes como valioso recurso natural. No entanto, devido à volatilidade dos óleos essenciais, o efeito de proteção se dissipa relativamente rápido. $O$ desenvolvimento de formulações capazes de manter os ingredientes ativos no alvo por mais tempo certamente ampliará a utilização destes óleos como repelentes (Nerio et al., 2009a).

Devido aos grandes males causados pela utilização em larga escala de agrotóxicos e inseticidas sintéticos, existe a necessidade de desenvolver novas tecnologias a fim de minimizar esta utilização. Além disso, há crescente interesse por produtos orgânicos, livres de agrotóxicos, bem como a conscientização de produtores e consumidores levando-os a atitudes ecologicamente corretas. Todos estes aspectos impulsionam a busca por produtos alternativos que não agridam o ambiente.

As plantas compõem rica fonte de pesquisa e muitos dos exemplares estudados mostraram-se promissores. No entanto, para a inserção definitiva e segura de produtos botânicos no mercado, estudos sobre mecanismos de ação, fitotoxidade, real segurança a mamíferos e outros vertebrados, entre outros assuntos, ainda são necessários.

\section{AGRADECIMENTO}

FAPESP e CNPq pelas bolsas concedidas; FUNDUNESP e PADC-FCF pelo suporte.

\section{REFERÊNCIA}

AHMED, B.I. et al. Field bioefficacy of plant extracts for the control of post flowering insect pests of cowpea (Vigna unguiculata (L.) Walp.) in Nigeria. Journal of Biopesticides, v.2, n.1, p.37-43, 2009.

BROUSSALIS, A.M. et al. Argentine plants as potential source of insecticidal compounds. Journal of Ethnopharmacology, v.67, p.219-23, 1999.

CARLINI, C.R.; GROSSI-DE-SÁ, M.F. Plant toxic proteins with insecticidal properties. A review on their potentialities as bioinsecticides. Toxicon, v.40, p.1515-39, 2002.

CARRANO-MOREIRA, A.F.; ALL, J. Screening of biopesticides against the cotton bollworm on cotton. Pesquisa Agropecuária Brasileira, v.30, n.3, p.307-12, 1995.

COELHO, M.B. et al. Insecticidal action of Annona coriacea lectin against the flour moth Anagasta kuehniella and the rice moth Corcyra cephalonica (Lepidoptera: Pyralidae). Comparative Biochemistry and Physiology, v.146, p.406-14, 2007.

COSTA, E.L.N. et al. Efeitos, aplicações e limitações de extratos de plantas inseticidas. Acta Biologica Leopoldensia, v.26, n.2, p.173-85, 2004.

DA SILVA, P. et al. Molecular requirements for the insecticidal activity of the plant peptide pea albumin 1 subunit $b$ (PA1b)S. The Journal of Biological Chemistry, v.285, n.43, p.32689-94, 2010.

DUGASSA, S. et al. Field investigation on the repellent activity of some aromatic plants by traditional means against Anopheles arabiensis and $A$. pharoensis (Diptera: Culicidae) around Koka, central Ethiopia. Acta Tropica, v.112, p.38-42, 2009.

EAMSOBHANA, P. et al. Laboratory evaluation of aromatic essential oils from thirteen plant species as candidate repellents against Leptotrombidium chiggers (Acari: Trombiculidae), the vector of scrub typhus. Experimental \& Applied Acarology, v.47, p.257-62, 2009.

GOVINDARAJAN, M. Larvicidal and repellent activities of Sida acuta Burm. F. (Family: Malvaceae) against three important vector mosquitoes. Asian Pacific Journal of Tropical Medicine, v.3, n.9, p.691-5, 2010.

HASHIM, M.S.; DEVI, K.S. Insecticidal action of the polyphenolic rich fractions from the stem bark of Streblus

Rev. Bras. PI. Med., Botucatu, v.13, n.4, p.500-506, 2011. 
asper on Dysdercus cingulatus. Fitoterapia, v.74, p.6706, 2003.

IRULANDI, S. et al. Effect of botanical insecticides on coffee berry borer, Hypothenemus hampei (Ferrari) (Coleoptera: Scolytidae). Journal of Biopesticides, v.1, n.1, p.70-3, 2008.

ISLAM, M.S. et al. Direct and admixture toxicity of diatomaceous earth and monoterpenoids against the storage pests Callosobruchus maculatus (F.) and Sitophilus oryzae (L.). Journal of Pest Science, v.83, p.105-12, 2010.

ISMAN, M.B. Plant essential oils for pest and disease management. Crop Protection, v.19, p.603-8, 2000.

JBILOU, R. et al. Insecticidal effects of extracts of seven plant species on larval development, a-amylase activity and offspring production of Tribolium castaneum (Herbst) (Insecta: Coleoptera: Tenebrionidae). Bioresource Technology, v.99, p.959-64, 2008.

KARUNAMOORTHI, K. et al. Laboratory evaluation of traditional insect/mosquito repellent plants against Anopheles arabiensis, the predominant malaria vector in Ethiopia. Parasitology Research, v.103, p.529-34, 2008. KIM, S.I. et al. Insecticidal activities of aromatic plant extracts and essential oils against Sitophilus oryzae and Callosobruchus chinensis. Journal of Stored Products Research, v.39, p.293-303, 2003.

KWEKA, E.J. et al. E.A. Ethnobotanical study of some of mosquito repellent plants in north-eastern Tanzania. Malaria Journal, v.7, p.152-60, 2008.

MALA, S.; MUTHALAGI S. Effect of neem oil extractive (NOE) on repellency, mortality, fecundity, development and biochemical analysis of Pericallia ricini (Lepidoptera: Arctidae). Journal of Biopesticides, v.1, n.1, p.63-6, 2008. MALARVANNAN, S. et al. Bioefficacy of crude and fractions of Argemone mexicana against tobacco caterpillar, Spodoptera litura Fab. (Noctuidae: Lepidoptera). Journal of Biopesticides, v.1, n.1, p.55-62, 2008.

MAO, L.; HENDERSON, G. Antifeedant activity and acute and residual toxicity of alkaloids from Sophora flavescens (Leguminosae) against formosan subterranean termites (Isoptera: Rhinotermitidae). Journal of Economic Entomology, v.100, n.3, p.866-70, 2007.

MENEZES, E.L.A. Inseticidas botânicos: seus princípios ativos, modo de ação e uso agrícola. Seropédica, Rio de Janeiro: Embrapa Agrobiologia, 2005. 58p.

MOORE, S.J. et al. Field evaluation of traditionally used plant-based insect repellents and fumigants against the malaria vector Anopheles darlingi in Riberalta, Bolivian Amazon. Journal of Medical Entomology, v.44, n.4, p.62430, 2007.

MURUGESAN, N.; MURUGESH. T. Efficacy of some plant products against spotted Leaf beetle (Hadda beetle),
Henosepilachna vigintiooctopunctata (F.) in Brinjal. Journal of Biopesticides, v.1, n.1, p.67-9, 2008.

NERIO, L.S. et al. Repellent activity of essential oils: A review. Bioresource Technology, 2009a. doi:10.1016/ j.biortech.2009.07.048

NERIO, L.S. et al. Repellent activity of essential oils from seven aromatic plants grown in Colombia against Sitophilus zeamais Motschulsky (Coleoptera). Journal of Stored Products Research, v.45, p.212-4, 2009b.

NOVO, J.P.S. et al. Pellets produzidos com estruturas de plantas e óleo essencial de folhas de Ageratum conyzoides I. Afetando o desenvolvimento de Sitophilus oryzae. Pesquisa Agropecuária Tropical, v.40, n.2, p.221-9, 2010.

PALSSON. K.; JAENSON, T.G.T. Plant products used as mosquito repellents in Guinea Bissau, West Africa. Acta Tropica, v.72, p.39-52, 1999.

PALUCH, G. et al. Quantitative structure-activity relationship of botanical sesquiterpenes: spatial and contact repellency to the yellow fever mosquito, Aedes aegypti. Journal of Agricultural and Food Chemistry, v.57, p.7618-25, 2009.

PAVELA, R. Insecticidal activity of certain medicinal plants. Fitoterapia, v.75, p.745-9, 2004.

RANI, U.P.; MURTHY, J.M. Botanical treatment for grain protection and their effects on seed germination and seedling performance of stored maize. Journal of Biopesticides, v.1, n.1, p.74-80, 2008.

RATHI, J.M. et al. Qualitative phytochemical screening of some locally available insecticidal plants. Journal of Biopesticides, v.1, n.1, p.52-4, 2008.

RAVIKUMAR, A. et al. Evaluation of certain organic nutrient sources against mealy bug, Coccidohystrix insolitus (Green.) and the spotted leaf beetle, Epilachna vigintioctopunctata Fab. on Ashwagandha, Withania somnifera Dunal. Journal of Biopesticides, v.1, n.1, p.2831, 2008.

SUJATHA, S. et al. Medicinal plants and its impact of ecology, nutritional effluents and incentive of digestive enzymes on Spodoptera litura (Fabricious). Asian Journal of Agricultural Research, v.4, n.4, p.204-11, 2010.

TONG, F.; COATS, J.R. Effects of monoterpenoid insecticides on $[3 \mathrm{H}]-\mathrm{TBOB}$ binding in house fly GABA receptor and $36 \mathrm{Cl}$ uptake in American cockroach ventral nerve cord. Pesticide Biochemistry and Physiology, v.98, p.317-24, 2010.

VASANTHARAJ, D.B. Biotechnological approaches in IPM and their impact on environment. Journal of Biopesticides, v.1, n.1, p.1-5, 2008.

WU, H.Q. et al. Acaricidal activities of traditional Chinese medicine against the house dust mite, Dermatophagoides farinae. Parasitology, v.137, p.975-83, 2010. 